\author{
Daniel Alonso* \\ Iván Kataryniuk* \\ Carlos Moreno* \\ Javier J. Pérez
}

\title{
EL PROGRAMA NEXT GENERATION EU: CARACTERÍSTICAS Y CLAVES PARA SU ÉXITO
}

\author{
El programa Next Generation EU se diseña para contribuir a impulsar el crecimiento \\ potencial, reducir las brechas entre economias y mitigar, temporalmente, dos carencias del \\ encaje institucional europeo (falta de elementos fiscales estabilizadores supranacionales \\ $y$ escasez de activos seguros). No obstante, su efecto final es incierto, y dependerá, \\ crucialmente, de la capacidad de asignar e implementar de manera transparente $y$ \\ eficiente los proyectos.
}

\section{The Next Generation EU program: features and keys to success}

The Next Generation EU program is designed to enhance potential growth, contribute towards the reductions of gaps between the European economies, and to mitigate, temporarily, two shortcomings in the European institutional framework (the lack of supranational stabilizing fiscal elements and the scarcity of safe assets). However, the final impact of the program is uncertain, and will depend crucially on the ability to allocate and implement in a transparent way the different projects.

Palabras clave: Next Generation EU, Mecanismo de Recuperación y Resiliencia, planes de recuperación y resiliencia, deuda pública, impacto económico.

Keywords: Next Generation EU, Recovery and Resilience Facility, recovery and resilience plans, public debt, economic impact.

JEL: E62, F43, F45, H54, H63.

\footnotetext{
* Banco de España.

Las opiniones vertidas en este artículo son de exclusiva responsabilidad

de los autores y no reflejan necesariamente las del Banco de España o el

Eurosistema.

Contacto: daniel.alonsosoto@bde.es; ivan.kataryniuk@bde.es;

carlos.moreno@bde.es; javierperez@bde.es

Versión de enero de 2022.

https://doi.org/10.32796/ice.2022.924.7356
} 


\section{Introducción}

El Consejo Europeo acordó el 21 de julio de 2020 la creación de un instrumento financiero de carácter temporal denominado Next Generation EU (NGEU), para financiar con recursos de la Unión Europea (UE) inversiones en los Estados miembros (EE MM) y promover la puesta en marcha de reformas económicas en áreas clave. EI NGEU persigue aumentar el potencial de crecimiento de la economía europea a medio y largo plazo, a través de un impulso reformador y de una reasignación de los recursos productivos hacia áreas como la digitalización o la lucha contra el cambio climático. Así pues, los objetivos del programa van más allá de compensar en el corto plazo las consecuencias económicas de la crisis, aunque ciertamente ha venido a suplir, temporalmente, la ausencia de un mecanismo de estabilización fiscal común en la arquitectura institucional de la UE.

El instrumento se financia con una emisión muy significativa de deuda pública europea supranacional, que está recibiendo una calificación crediticia del máximo nivel. Esto también es una novedad importante en el marco de la UE, de manera que el NGEU supone un impulso importante para aumentar la disponibilidad de activos en euros que pueden considerarse seguros. La deuda se amortizará a partir de 2028 con cargo a instrumentos impositivos comunes de la UE, para lo cual se ha definido una hoja de ruta para aumentar los recursos propios de la Comisión Europea (CE).

EI NGEU es, por tanto, un paso muy importante en la integración europea ya que ha contribuido a mitigar, al menos temporalmente, dos carencias fundamentales del encaje institucional europeo: la falta de elementos fiscales estabilizadores supranacionales y la escasez relativa de activos seguros en euros.

La falta de precedentes de programas de esta ambición, no obstante, hace que exista una elevada incertidumbre sobre la capacidad transformadora del NGEU sobre las economías de la UE y la UE en su conjunto. Si bien las estimaciones contrafactuales disponibles son prometedoras sobre el potencial impacto del NGEU (véase Pfeiffer et al., 2021, entre otros), su efecto final dependerá, crucialmente, de una serie de cuestiones, entre las que destacamos las siguientes: ¿Serán capaces los agentes económicos (gobiernos, empresas, familias) de implementar de acuerdo con los calendarios previstos los proyectos comprometidos? ¿Será esta aplicación eficiente (en particular desde el punto de vista de su gestión administrativa)? ¿Se verá mitigado su impacto por posibles cuellos de botella en el suministro de insumos? ¿Será suficiente la cuantía aprobada para conseguir una transformación permanente de la UE? ¿Cuáles serán los impactos económicos finales sobre el crecimiento potencial? ¿Divergirán o convergerán las economías de la UE por la acción del instrumento? ¿Producirá el NGEU efectos crowding-out significativos sobre la actividad privada dado que su aplicación es probable que se realice, en gran medida, en un contexto de expansión económica generalizada en la UE? Las respuestas a estas preguntas se irán resolviendo en los próximos meses y años, para lo cual será necesario un esfuerzo significativo de transparencia por parte de las distintas Administraciones públicas, tanto nacionales como supranacionales, en particular en lo relativo a la publicación y diseminación de toda la información necesaria, para que los expertos, y la sociedad en su conjunto, puedan monitorizar la implementación de los planes y evaluar su impacto económico y social.

El resto del artículo profundiza en estas cuestiones. En primer lugar, en el apartado 2 se describen las principales características del NGEU —estructura, recursos, financiación, heterogeneidad de los planes de recuperación y resiliencia (PRR), aspectos de la gobernanza de estos, comunes y nacionales-. Por su parte, en el apartado 3 se describe la situación del despliegue del instrumento hasta finales de 2021. El apartado 4 adopta un tono más normativo y revisa los condicionantes de la capacidad transformadora que acabe teniendo el NGEU, siguiendo el hilo de las preguntas enunciadas anteriormente. Finalmente, en el apartado 5 se proporcionan las principales conclusiones del estudio. 


\section{FIGURA 1}

\section{RESUMEN DE LOS INSTRUMENTOS VINCULADOS AL NGEU Y AL PRESUPUESTO EUROPEO}
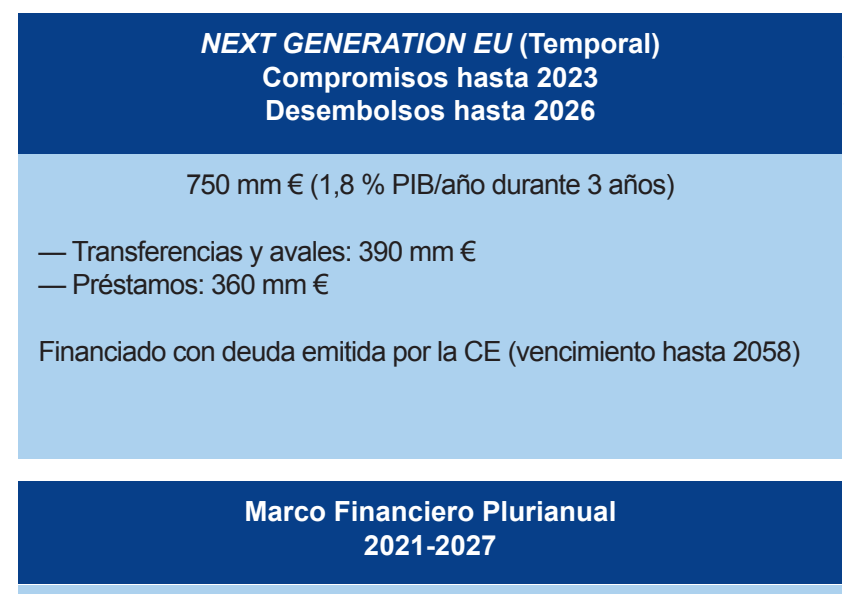

$1.074 \mathrm{~mm} €$

(1,1 \% PIB/año durante 7 años)

Financiado con contribuciones de los EE MM

\section{Principales características del NGEU}

\section{Estructura y recursos}

El programa NGEU está compuesto principalmente por dos bloques con un montante de recursos totales, que se deben comprometer en 2021-2023, que asciende a $750 \mathrm{mil}$ millones $(\mathrm{mm})$ de euros, 1,8 puntos porcentuales (pp) del PIB de la UE por año (véase Figura 1). El primer bloque, al que se destina el $10 \%$ de los fondos del NGEU, se centra en programas paneuropeos y en programas de acción rápida en la lucha contra la pandemia entre los que destaca el REACT-EU (Recovery Assistance for Cohesion and the Territories of Europe) dotado con 47,5 mm de euros,

NOTA: $\mathrm{mm}=$ mil millones.

FUENTE: Elaboración propia a partir de información de la Comisión Europea.
FONDOS PARA PROGRAMAS NACIONALES

(Inversión y reformas estructurales)

Total: $672,5 \mathrm{~mm} €(1,6 \%$ PIB/año)

MECANISMO DE RECUPERACIÓN Y RESILIENCIA

(Ligado al Semestre Europeo)

- Transferencias: $312,5 \mathrm{~mm} €(0,75 \% \mathrm{PIB} / \mathrm{año})$

$70 \%$ comprometido en 2021-2022

$30 \%$ comprometido en 2023

—Préstamos: $360 \mathrm{~mm} €(0,85 \%$ PIB/año $)$

\section{FONDOS PARA PROGRAMAS PANEUROPEOS} Total: $77,5 \mathrm{~mm} €(0,2 \%$ PIB/año)

— REACT-EU: Desembolso acelerado fondos de cohesión Recursos: $47,5 \mathrm{~mm} €$

— InvestEU: Refuerzo del antiguo Plan Juncker Recursos: $5,6 \mathrm{~mm} €$

— Otros fondos: Ayuda a la innovación e investigación, transición ecológica, desarrollo rural, catástrofes Recursos: $24,4 \mathrm{~mm} €$ y cuyos recursos deberán ser ejecutados en los dos primeros años del programa (2021-2022) (Comisión Europea, 2020a) ${ }^{1}$.

El segundo bloque, el Mecanismo de Recuperación y Resiliencia (MRR), absorberá el $90 \%$ de los recursos del programa, estando dotado con hasta $672,5 \mathrm{~mm}$ de euros para apoyar inversiones y reformas en el período 2021-2026. Este total se divide en transferencias directas no reembolsables de la CE a los EE MM, por valor máximo de $312,5 \mathrm{~mm}$ de euros, y en préstamos bilaterales de la CE a los EE MM que ascenderían, de ser

\footnotetext{
1 Además del REACT-EU, existen varios programas de menor cuantía: Fondo de Transición Justa, Desarrollo Rural, InvestEU, rescEU y Horizonte Europa.
} 
utilizados en su totalidad, a $360 \mathrm{~mm}$ de euros ${ }^{2}$, y que tendrán que ser devueltos por estos una vez finalice la fase de inversiones y reformas, como se precisará más adelante. Para acceder a la financiación del MRR, cada EE MM debía presentar a la CE un PRR, que especifique las inversiones y reformas que financiará con los fondos para alcanzar los objetivos del MRR dentro del período de referencia del mismo.

La asignación de los recursos del MRR entre los EE MM sigue unas reglas que vienen definidas en el Reglamento correspondiente (Unión Europea, 2021). Por una parte, en cuanto al componente de préstamos, los países pueden realizar sus solicitudes hasta agosto de 2023 , por un volumen máximo del $6,8 \%$ de su renta nacional bruta (RNB) en 2019 a precios corrientes, no habiendo cuantías preasignadas a los EE MM. Bajo el supuesto de que solo solicitaran préstamos del MRR los países que, durante la pandemia, han accedido a préstamos de la UE en el marco del Instrumento Europeo de Apoyo Temporal para Mitigar los Riesgos de Desempleo en una Emergencia (SURE, por sus siglas en inglés) ${ }^{3}$, el monto máximo asignado sería cercano a los $380 \mathrm{~mm}$ de euros, cifra muy próxima a la dotación total destinada a préstamos del programa.

Por su parte, el componente de transferencias directas del MRR se determina de acuerdo con unos criterios que generan una significativa heterogeneidad en las cuantías a recibir por los países, tanto en términos absolutos como en porcentaje de su PIB (véase la Figura 2). Estos criterios toman en consideración tanto la merma económica sufrida durante la crisis derivada de la pandemia (un $30 \%$ del total), ponderando la caída del PIB en 2020 y la pérdida acumulada de producto en 2020-2021, como las diferencias

\footnotetext{
${ }^{2}$ Estas cifras se refieren a precios de 2019. El montante en precios corrientes es ligeramente superior.

${ }^{3}$ EI SURE proporcionó ayuda financiera a los EE MM, hasta un máximo de $100 \mathrm{~mm}$ de euros, para hacer frente al aumento del gasto público resultante de los esquemas de protección del empleo aprobados durante la pandemia. A diferencia de los préstamos del MRR, cuyo respaldo financiero descansa en el presupuesto de la UE, los préstamos del SURE están respaldados por garantías de los EE MM.
}

estructurales entre los EE MM (el $70 \%$ restante), ponderadas con respecto al agregado de la UE-27, en PIB per cápita nominal (en 2019), PIB real (en 2019-2021), RNB per cápita (en 2019), población (en 2019) y tasa de paro (en 2015-2019). La traducción de estas variables en una asignación de fondos por países se realiza de acuerdo con las fórmulas presentadas en el Anexo IV del Reglamento del MRR.

Las cuantías provisionales actualmente asignadas, por tanto, dependen de las estimaciones disponibles para 2020 y 2021. En términos prácticos, la caída en el PIB real en 2020 y la pérdida acumulada de producto en el período 2020-2021 se estimaron inicialmente tomando como base las previsiones de otoño 2020 de la CE. No obstante, las asignaciones definitivas se actualizarán el 30 de junio de 2022, momento en el que Eurostat publicará su primera estimación del PIB de los EE MM en 2021. A modo de ilustración, en la Figura 3 se presentan estimaciones de los fondos MRR que recibirían las cuatro principales economías de la UE (Alemania, Francia, Italia y España), Grecia y Portugal, usando previsiones alternativas asociadas a dos momentos del tiempo: la fecha en la que se propuso inicialmente el NGEU (mayo de 2020) y un período más reciente (julio de 2021). En la figura se observa, por ejemplo, que, como consecuencia del mayor impacto sobre la actividad económica de la pandemia estimado en julio de 2021, con respecto al que se preveía a mitad de 2020, España recibiría una mayor cantidad adicional de fondos de lo que se habría previsto inicialmente.

\section{Financiación}

Para financiar el NGEU, la CE planea emitir deuda por valor de alrededor de $800 \mathrm{~mm}$ de euros en el período 2021-2026. Por primera vez en su historia, la CE está utilizando una estrategia de financiación diversificada, similar a la del Mecanismo Europeo de Estabilidad (MEDE). Bajo este método, la CE emite valores a diferentes plazos, con el objetivo de optimizar 
FIGURA 2

ASIGNACIONES DEL MECANISMO DE RECUPERACIÓN Y RESILIENCIA (MRR) POR PAÍS (En \%)

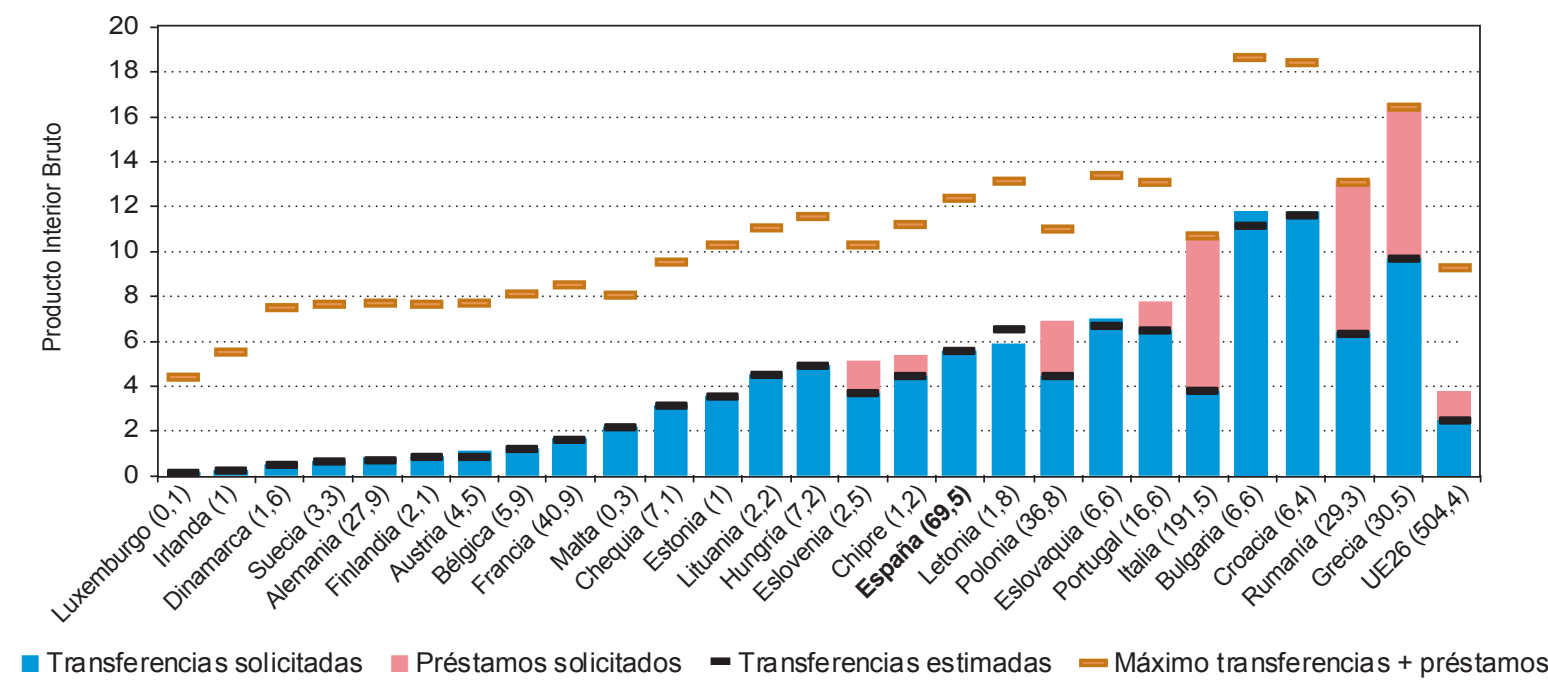

NOTA: Esta figura muestra las transferencias y préstamos del MRR solicitados por los países en sus PRR, así como las transferencias y préstamos estimados que corresponderían a cada país como porcentaje del PIB de 2019. Las transferencias del MRR estimadas proceden del Anexo IV del Reglamento sobre MRR, que se basa en las previsiones de otoño de 2020 de la Comisión Europea. Según el Reglamento, el importe final de las subvenciones se calculará a mediados de 2022. Préstamos MRR máximos: 6,8 \% de la renta nacional bruta de 2019 a precios corrientes en euros. Entre paréntesis se muestran los montantes totales de transferencias y préstamos solicitados por cada país en la fecha de cierre de este artículo (en miles de millones de euros).

FUENTE: Elaboración propia a partir de Eurostat y Comisión Europea (2020b).

el coste de las emisiones. Esta estrategia también se aplica a los préstamos del MRR, cuyo coste refleja el tipo de interés medio del conjunto de las emisiones. Este método sustituye a la financiación back to back, que caracterizaba al resto de préstamos de la UE a los EE MM, y por la que estos estaban vinculados (en coste y duración) a la financiación obtenida de forma paralela por la UE en los mercados.

Entre 2028 y 2058 la UE debe reembolsar la deuda contraída en los mercados financieros. La parte del MRR distribuida como transferencias se pagará a través del presupuesto de la UE, con los ingresos que se obtengan de la creación de nuevas figuras impositivas $y$, si fuera necesario, con unas mayores contribuciones al presupuesto común por parte de todos los EE MM. Asimismo, los países que hayan accedido a préstamos del MRR comenzarán los reembolsos 10 años después de la fecha del desembolso inicial, amortizando el principal en 20 pagos anuales iguales ${ }^{4}$.

Para garantizar que la UE pueda cubrir todos los pasivos resultantes del NGEU, como garantía, la UE ha aumentado el importe máximo que puede requerir a los EE MM en forma de contribuciones al presupuesto de la UE en 0,6 puntos porcentuales más de la RNB de la UE, hasta el $2 \%$ de la misma. La necesidad de recurrir a esta asignación adicional será solo temporal, ya que las obligaciones financieras y los pasivos contingentes relevantes disminuirán con el tiempo a medida que se reembolsen los fondos prestados.

\footnotetext{
${ }^{4}$ Véase https://www.europarl.europa.eu/doceo/document/E-9-2021003465-ASW_EN.html
} 


\section{FIGURA 3}

\section{REPARTO HIPOTÉTICO DE LAS TRANSFERENCIAS DEL MECANISMO DE RECUPERACIÓN Y RESILIENCIA USANDO LAS PREVISIONES DE LA COMISIÓN EUROPEA DE MAYO 2020 Y DE JULIO 2021}

Asignaciones transferencias Mecanismo de Recuperación y Resiliencia

Miles de millones

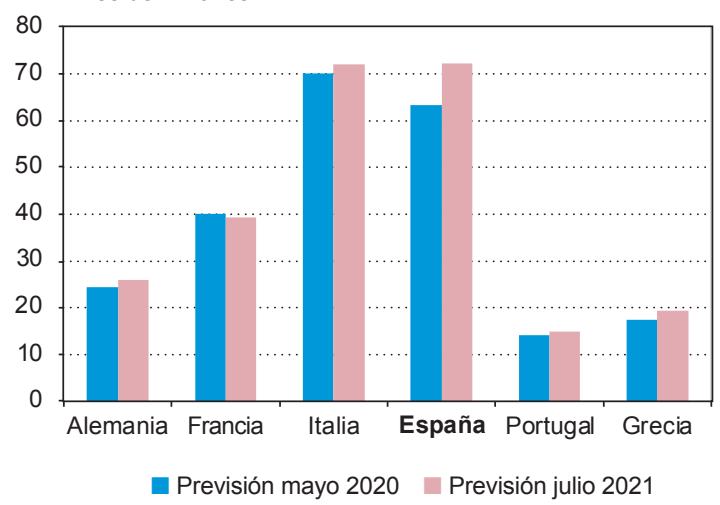

Cambio en las asignaciones como \% del total (2020 vs. 2021)

Puntos porcentuales

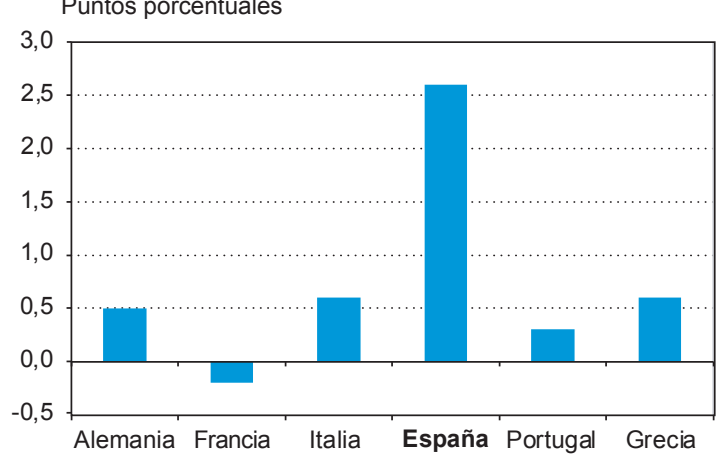

NOTA: El panel de la izquierda muestra las transferencias estimadas (en miles de millones de euros) utilizando la fórmula del Anexo IV del Reglamento del MRR, según las previsiones de la Comisión Europea de mayo de 2020 (cuando se estaba diseñando el mecanismo) y las previsiones de julio de 2021. El panel de la derecha muestra la diferencia (en puntos porcentuales) entre el porcentaje sobre el total que suponen las transferencias estimadas de cada país según las previsiones de julio de 2021 y las previsiones de mayo de 2020.

FUENTE: Elaboración propia a partir de Eurostat y Unión Europea (2021).

Como se ha mencionado anteriormente, junto con la negociación del MRR se debatió y acordó una hoja de ruta para introducir nuevos recursos propios para la CE . $^{5}$. El primer componente es una nueva contribución nacional calculada sobre la base de los residuos de envases de plástico no reciclados. Por otra parte, la CE presentó una propuesta en julio de 2021 para un mecanismo de ajuste en frontera de las emisiones de carbono (CBAM, por sus siglas en inglés) y una reforma del sistema de comercio de derechos de emisión de la UE (ETS, por sus siglas en inglés), por la cual a la CE le corresponderían un $25 \%$ de los ingresos obtenidos por las subastas de derechos. Además, en diciembre de 2021 la CE propuso que los EE MM contribuyan al presupuesto europeo por un $15 \%$ de la participación en los beneficios

\footnotetext{
${ }^{5}$ Véase Decisión (UE, Euratom) 2020/2053 del Consejo de 14 de diciembre de 2020 en https://www.boe.es/boe/dias/2021/06/11/pdfs/ BOE-A-2021-9720.pdf
}

de las multinacionales reasignados a los EE MM, en virtud del acuerdo OCDE/G20 de octubre de 2021 en el marco del Inclusive Framework on Base Erosion and Profit Shifting (BEPS) sobre la reasignación de los derechos de imposición de las multinacionales en función del lugar de procedencia de sus clientes finales. Finalmente, para junio de 2024, se espera que la CE proponga nuevos recursos propios adicionales (D'Alfonso et al., 2021), que podrían entrar en vigor el 1 de enero de 2026.

\section{La gobernanza del MRR}

\section{Los PRR y el papel de la Comisión Europea}

Los PRR nacionales se han realizado siguiendo una serie de criterios, que son los que utiliza la $C E$ para aprobarlos y evaluar su cumplimiento. La aprobación general de un plan por parte de la CE conlleva el desembolso de una prefinanciación (de hasta 
el $13 \%$ del total), con el objetivo de facilitar un despliegue rápido de los fondos. El resto de desembolsos seguirá un calendario propio de cada país, vinculado al logro de los hitos y objetivos fijados en el propio PRR nacional.

Los criterios sirven para orientar la composición del gasto de las acciones incluidas en los PRR, así como la definición de las reformas a abordar. En cuanto al gasto previsto en los PRR, los países deben alinearlo, a su vez, con los seis pilares generales que se han fijado: i) transición verde; ii) transformación digital; iii) crecimiento inteligente, sostenible e integrador, incluida la cohesión económica, el empleo, la productividad, la competitividad, la investigación, el desarrollo y la innovación, y un mercado único que funcione correctamente con pymes fuertes; iv) cohesión social y territorial; v) salud y resiliencia económica, social e institucional; y vi) políticas para la próxima generación, niños y jóvenes, incluida la educación y las habilidades. Los PRR deben asignar al menos el $37 \%$ de los fondos al objetivo i) y al menos el $20 \%$ al ii).

En lo referido a las reformas, estas deben abordar eficazmente las orientaciones de política económica identificadas en el marco del Semestre Europeo, conocidas como recomendaciones específicas de país ${ }^{6}$, así como la implementación del pilar europeo de derechos sociales. Asimismo, la liberación por parte de la CE de los desembolsos asociados a los PRR se encuentra vinculada al cumplimiento por parte del Estado miembro del marco de reglas fiscales. Así, en su artículo 10, el Reglamento señala que, en el caso de que el Consejo decida que un Estado miembro no ha tomado medidas efectivas para corregir su déficit excesivo, la CE podrá presentar una propuesta al Consejo para suspender la totalidad o parte de los compromisos o pagos.

\footnotetext{
${ }^{6}$ El Reglamento especifica que deben cumplirse las recomendaciones de 2019 y de 2020. Mientras que las de 2019 trataban sobre dificultades estructurales de las economías, las de 2020 estaban focalizadas en los instrumentos para hacer frente a la pandemia. Para una consulta sobre las recomendaciones específicas relevantes para esta evaluación, véase García Perea et al. (2020).
}

\section{La heterogeneidad de los PRR y su seguimiento}

Aunque los requisitos, criterios y prioridades para la elaboración de los PRR son comunes para todos los EE MM, los planes específicos muestran diferencias significativas en varios aspectos.

El primer aspecto es el número de hitos y objetivos (las condiciones) que establece cada país para alcanzar los objetivos marcados por la $\mathrm{CE}^{7}$. En general, los países que más fondos reciben en términos relativos han planificado un mayor número de hitos. Además, los hitos y objetivos también difieren en función de si la financiación se proporciona vía transferencias o préstamos, siendo en este último caso mucho menor la condicionalidad, al requerirse muchos menos hitos para cumplir los objetivos.

Un segundo aspecto en el que difieren los PRR de los EE MM es en el calendario de pagos (véase Figura 4). Italia, por ejemplo, ha distribuido las fechas de recepción esperada de fondos de manera muy uniforme en el tiempo, de forma que espera recibir pagos de tamaños similares cada seis meses. Otros países, como España, han planeado en cambio una ejecución anticipada, de manera que se concentran los pagos al inicio del período de ejecución del programa (segunda mitad de 2021 y principios de 2022). Por su parte, algunos países menos beneficiados por el programa, como Francia o Alemania, esperan recibir pagos más espaciados en el tiempo.

Los PRR nacionales también difieren en el foco (composición) de las inversiones previstas (véase Figura 5). Si bien algunos países, como Grecia o Portugal, han diversificado sus esfuerzos de inversión relacionados con el MRR más allá de las áreas verde y digital, otros países como Alemania parecen haber concentrado sus esfuerzos en una gama más reducida de inversiones, en particular, las relacionadas con la digitalización. La comparativa de los diferentes planes presentados sugiere que un área prioritaria en

\footnotetext{
${ }^{7}$ Esta condicionalidad está, en todo caso, vinculada a mejorar el potencial de crecimiento de las economías. Véase Arce et al. (2020).
} 


\section{FIGURA 4}

\section{CALENDARIO DE PAGOS SEGÚN LOS PLANES DE RECUPERACIÓN Y RESILIENCIA NACIONALES \\ (En \% del PIB)}
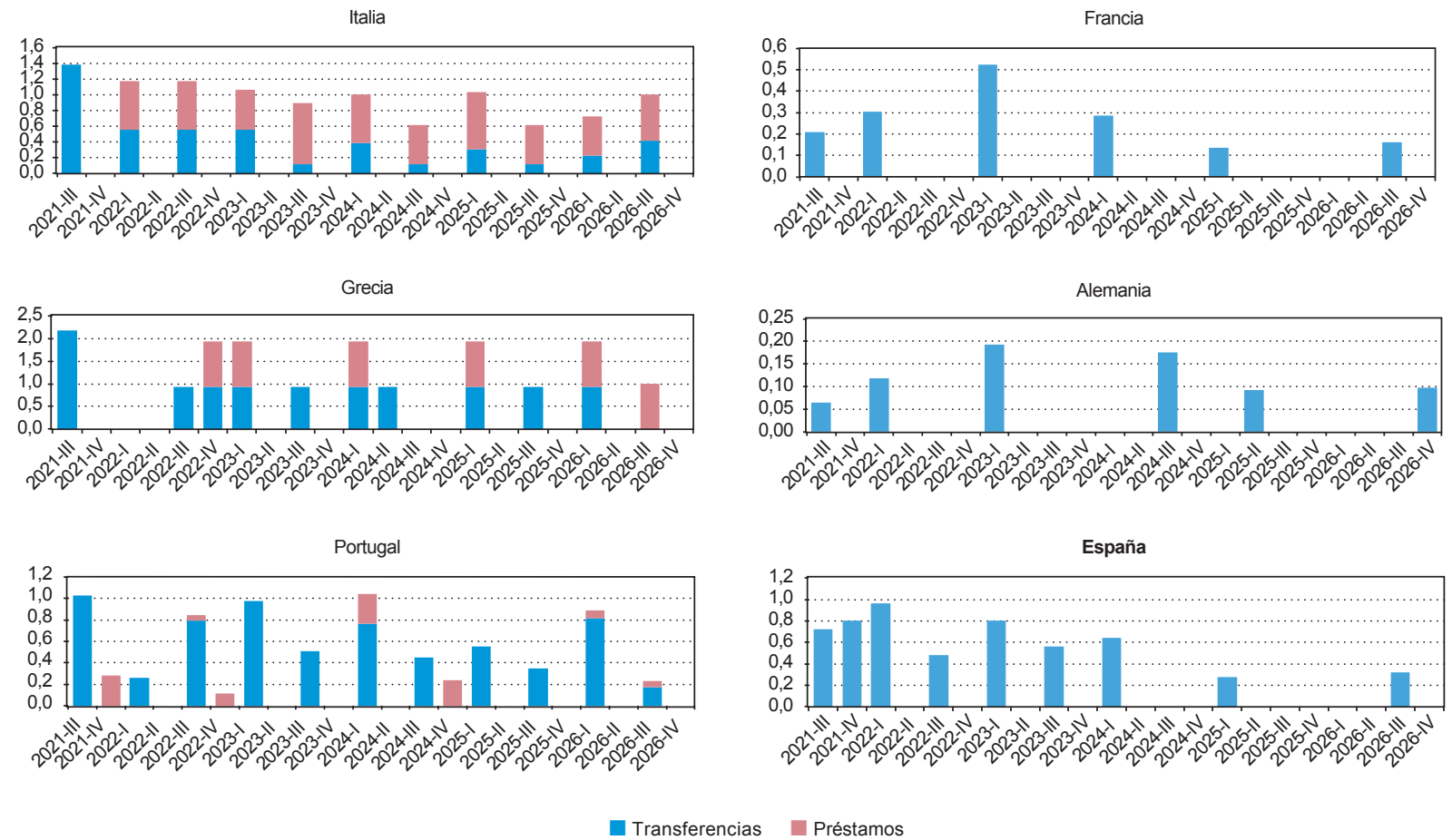

NOTA: Esta figura muestra el calendario trimestral de pagos de transferencias y préstamos (en \% del PIB) según se reportan en los planes de recuperación y resiliencia nacionales. Para estimar el trimestre en el que se recibe un pago se utiliza la fecha de cumplimiento del último hito/objetivo necesario para cumplir con dicho pago. Se asume además que el pago lo realiza la Comisión Europea en el trimestre siguiente a dicha fecha.

FUENTE: Banco de España a partir de Comisión Europea (2021b).

varios EE MM es la sostenibilidad y la movilidad inteligente, con un enfoque en la renovación de edificios para la eficiencia energética y las estaciones de carga de vehículos eléctricos. Alemania, por ejemplo, planea apoyar la producción masiva de hidrógeno y la inteligencia artificial. Varios países prevén acometer proyectos a gran escala, como infraestructura de transporte, combinándolos con los Fondos de Cohesión. Adicionalmente, los países han planteado invertir en formación para la adaptación de los mercados laborales a los retos climáticos y digitales.
Dentro de esta significativa heterogeneidad, en promedio, para las cuatro mayores economías de la zona euro, Portugal y Grecia, el 30 \% del gasto se prevé que se destinará a inversión digital (superando el $20 \%$ establecido por la CE) y el $42 \%$ a inversión verde (superando también el mínimo del 37 \%). Además, los PRR están alineados con las prioridades fijadas por la CE. El 41 \% de la inversión verde se destina al transporte sostenible y estaciones de carga, el $19 \%$ a tecnologías limpias y renovables y el $26 \%$ a la eficiencia energética de edificios. Solo el 


\section{FIGURA 5}

\section{COMPOSICIÓN DE LAS INVERSIONES EN LOS PRR}

(En \% total PRR)

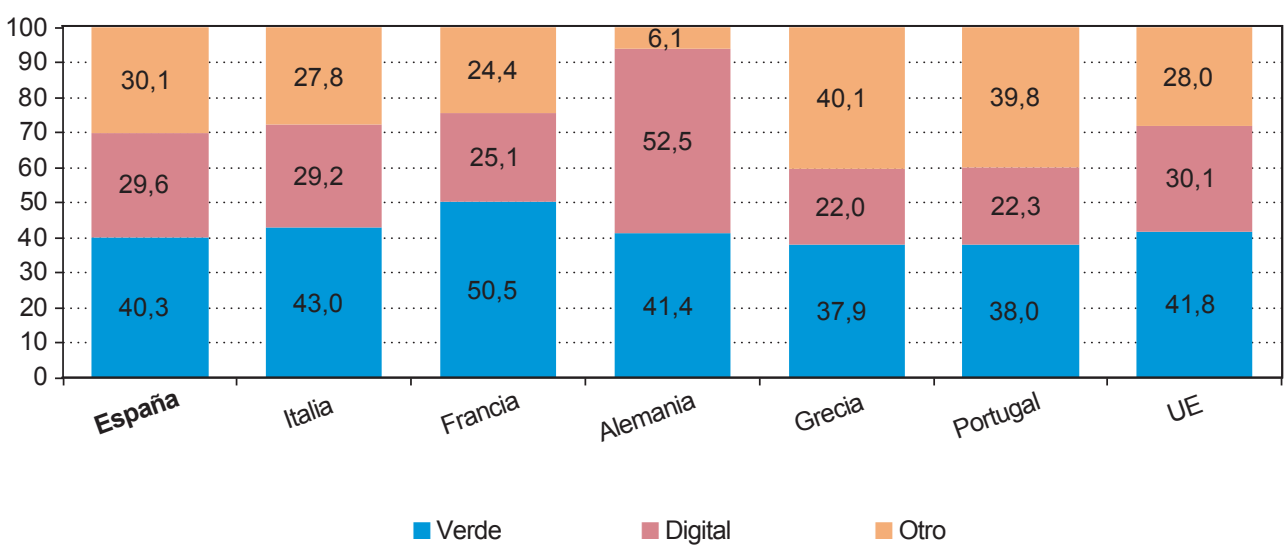

NOTA: Esta figura muestra el \% del total de las inversiones destinado a la transición digital, verde y a otras partidas según lo detallado en los planes de recuperación y resiliencia. La UE muestra los países disponibles.

FUENTE: Bruegel (2021)

$14 \%$ se destina a otros objetivos ecológicos fuera de las áreas prioritarias. Igualmente, la inversión digital en los PRR se alinea bien con las cuatro prioridades digitales establecidas por la CE (Comisión Europea, 2021a). En promedio, para los seis EE MM tomados como ejemplo, el $36 \%$ de la inversión digital se destinará a la educación y formación para apoyar las habilidades digitales, el $36 \%$ a la digitalización de la Administración pública, el $17 \%$ a mejorar los servicios rápidos de banda ancha, el $4 \%$ para capacidades de nube de datos y procesadores sostenibles y el $6 \%$ para otras inversiones digitales.

Un último aspecto diferenciador de los PRR nacionales que cabe resaltar es que los planes deben abordar las recomendaciones específicas realizadas por la CE a cada país (véanse Tabla 1 y Anexo). En promedio, los PRR presentados hasta la fecha las abordan en un $96 \%$ (85\% «significativamente» $y$ $11 \%$ «parcialmente»). No obstante, siete EE MM no abordan todas las recomendaciones en sus planes y tres de ellos no abordan más del $10 \%$ de las mismas. En relación con las reformas planteadas, estas se centran en aspectos como la simplificación administrativa (Bélgica, Grecia o Alemania), la reforma de la Administración de Justicia (Grecia o Italia) o la sostenibilidad de las finanzas públicas mediante la elaboración de revisiones de gasto, reformas de los sistemas de pensiones o del marco impositivo (Bélgica, España, Italia o Rumanía).

\section{Estructuras de gobernanza nacionales}

A nivel nacional, las estructuras de gobernanza y control establecidas por los Estados en los PRR comparten una estructura básica que consta de tres niveles. Normalmente el Ministerio de Hacienda y/o Economía es el principal responsable de los planes y se sitúa en el primer nivel (estratégico y de coordinación) a veces junto con otros ministerios. En un segundo nivel (de coordinación técnica y monitorización), se encuentran 
TABLA 1

\section{RECOMENDACIONES EN LOS PRR}

\begin{tabular}{|c|c|c|c|c|c|c|c|c|}
\hline & $\begin{array}{c}\text { Total } \\
\text { recomendaciones }\end{array}$ & No cubiertas & $\begin{array}{l}\text { Parcialmente } \\
\text { cubiertas }\end{array}$ & $\begin{array}{c}\text { Significativamente } \\
\text { cubiertas }\end{array}$ & $\begin{array}{c}\text { No cubiertas } \\
(\%)\end{array}$ & $\begin{array}{l}\text { Parcialmente } \\
\text { cubiertas (\%) }\end{array}$ & $\begin{array}{l}\text { Significativamente } \\
\text { cubiertas (\%) }\end{array}$ & $\begin{array}{l}\text { Nombre de las } \\
\text { no cubiertas }\end{array}$ \\
\hline Austria & 10 & 0 & 2 & 8 & 0 & 20 & 80 & \\
\hline Bélgica & 17 & 3 & 1 & 13 & 18 & 6 & 76 & $\begin{array}{l}\text { Mejor coordinacón de } \\
\text { las politicas fiscales; } \\
\text { sostenibilidad de } \\
\text { la deuda pública; } \\
\text { competencia en } \\
\text { servicios }\end{array}$ \\
\hline Croacia & 16 & 0 & 0 & 16 & 0 & 0 & 100 & \\
\hline Chipre & 9 & 0 & 0 & 9 & 0 & 0 & 100 & \\
\hline $\begin{array}{l}\text { República } \\
\text { Checa }\end{array}$ & 13 & 0 & 2 & 11 & 0 & 15 & 85 & \\
\hline Dinamarca & 12 & 3 & 2 & 7 & 25 & 17 & 58 & $\begin{array}{l}\text { Educación y } \\
\text { capacidades; acciones } \\
\text { contra el blanqueo de } \\
\text { dinero }\end{array}$ \\
\hline Francia & 20 & 1 & 2 & 17 & 5 & 10 & 85 & $\begin{array}{l}\text { Complejo sistema de } \\
\text { pensiones }\end{array}$ \\
\hline Alemania & 14 & 1 & 2 & 11 & 7 & 14 & 79 & $\begin{array}{l}\text { Competencia en } \\
\text { servicios empresariales } \\
\text { y profesiones reguladas }\end{array}$ \\
\hline Grecia & 19 & 0 & 2 & 17 & 0 & 11 & 89 & \\
\hline Irlanda & 16 & 1 & 3 & 12 & 6 & 19 & 75 & Ampliación base fiscal \\
\hline Italia & 12 & 0 & 1 & 11 & 0 & 8 & 92 & \\
\hline Letonia & 16 & 0 & 4 & 12 & 0 & 25 & 75 & \\
\hline Lituania & 17 & 0 & 2 & 15 & 0 & 12 & 88 & \\
\hline Luxemburgo & 16 & 2 & 1 & 13 & 13 & 6 & 81 & $\begin{array}{l}\text { Afrontar planes fiscales } \\
\text { agresivos; reducir } \\
\text { las barreras a la } \\
\text { competición }\end{array}$ \\
\hline Portugal & 16 & 1 & 2 & 13 & 6 & 13 & 81 & Interconexión energética \\
\hline Eslovaquia & 8 & 0 & 0 & 8 & 0 & 0 & 100 & \\
\hline Eslovenia & 12 & 0 & 0 & 12 & 0 & 0 & 100 & \\
\hline España & 21 & 0 & 6 & 15 & 0 & 29 & 71 & \\
\hline
\end{tabular}

NOTA: La mayoría de planes presentados abordan todas las recomendaciones específicas de cada país (parcial o significativamente). Únicamente siete planes no abordan alguna recomendación y solo tres de ellos no abordan más del $10 \%$ de las recomendaciones (Bélgica, Dinamarca y Luxemburgo).

FUENTE: Elaboración propia a partir de Comisión Europea (2021c).

los comités o agencias encargados de la implementación y supervisión de los planes, en el que los ministerios competentes también suelen participar, en particular en la evaluación de hitos y objetivos. En un tercer nivel, las instituciones de auditoría nacionales o un comité creado para tal fin se encargan del control interno de los planes. Finalmente, algunos países, como Italia y Portugal, han planteado una estructura de carácter horizontal, en el que 
grupos de interés y expertos externos participan en la implementación, y a veces en la evaluación, de los planes.

En cualquier caso, la naturaleza y el marco de estructuras de gobernanza vienen muy influidos por la propia estructura política del Estado miembro (más o menos centralizada, federal o unitaria) y, por tanto, estas difieren por país. Por ejemplo, en algunos países las autoridades locales y regionales tienen un papel relevante, principalmente en la implementación de los planes (segundo nivel), como en el caso de España.

Dentro de este esquema general, los EE MM han utilizado estructuras de gobernanza establecidas 0 han creado otras nuevas. Grecia e Italia son ejemplos de estructuras de gobernanza nuevas, establecidas específicamente para garantizar la implementación y el seguimiento adecuados del PRR, a pesar de que siguen dependiendo de las estructuras y órganos existentes para tareas específicas. En el caso de Grecia, un organismo se dedica a la supervisión de las reformas y se prevé que estas sean certificadas por auditores independientes. En Italia, un organismo específico tiene el cometido de facilitar la eliminación de obstáculos administrativos para la implementación del plan. En España y Portugal se están creando estructuras adicionales, en particular para la orientación y el control políticos. Algunas de las estructuras que se utilizan a nivel nacional son las que actualmente se encargan de gestionar otros fondos de la UE o seguirán procedimientos similares (como en el caso de España).

Varios hitos en los planes nacionales abordan los sistemas de gobernanza. Este es el caso, en particular, de los planes francés, griego e italiano. Estos hitos deben cumplirse antes de que los respectivos Estados miembros realicen la primera solicitud de pago. En algunos casos, los hitos se relacionan con cambios legislativos; en otros, también para garantizar que se desplegará suficiente capacidad administrativa para garantizar un seguimiento adecuado (es el caso, por ejemplo, de Grecia).

En el caso del esquema de gobernanza español, en el primer nivel, el diseño y la supervisión son llevados a cabo por la Comisión de Recuperación, Transformación y Resiliencia, presidida por el presidente del Gobierno, y formada por ministros y secretarios de Estado. La implementación será realizada por la Secretaría General de Fondos Europeos dentro del Ministerio de Hacienda, que es la autoridad responsable del plan frente a la Comisión Europea, e integra tanto a nuevas estructuras para la gestión del MRR como a las estructuras previas encargadas de la gestión de los fondos europeos. Esta Secretaría General es la encargada de las peticiones para las reclamaciones de pago que estén sujetas al logro de los objetivos. Finalmente, en el tercer nivel, la Intervención General de la Administración del Estado (IGAE) certificará el logro de los objetivos. Como se ha comentado anteriormente, no se prevé una estructura formal en la que se consulte a agentes externos sobre la implementación del PRR. No obstante, se ha establecido un organismo de cooperación entre la Administración central y las locales y regionales, la Conferencia Sectorial del Plan de Recuperación, y, además, el Gobierno deberá informar cada semestre del progreso del plan al Parlamento a través de la Comisión Mixta para la Unión Europea, así como mantener informados a los agentes sociales.

\section{Los datos para el seguimiento de los planes}

La implementación de un programa del tamaño y la complejidad del NGEU requiere, como se ha discutido anteriormente, de estructuras de gobernanza adecuadas, pero también de la disponibilidad de datos de publicación frecuente, de calidad y con la suficiente granularidad. La disponibilidad de información permitiría realizar un seguimiento cercano de la adecuación de compromisos y realidades que ancle de manera objetiva la liberación de los fondos por parte de la CE. Asimismo, la transparencia en todas las fases del proceso permitirá generar el suficiente grado de ownership en los países de la UE y la sociedad europea en su conjunto. 
Conscientes de este reto, las autoridades han diseñado, entre otros instrumentos, el Recovery and Resilience Scoreboard ${ }^{8}$, para diseminar toda la información disponible sobre la implementación del NGEU. La información disponible, con carácter general, se puede agrupar en dos bloques. Por una parte, la información compilada por la CE sobre la implementación de los PRR, en base a los datos enviados por los EE MM sobre el cumplimiento de hitos y objetivos, y sobre los desembolsos realizados. La CE también ha desarrollado indicadores propios de gasto realizado, siguiendo una metodología transparente ${ }^{9}$. Por otra parte, un segundo bloque de indicadores es el que se comprometen a compilar los EE MM relativos a la implementación de los planes ${ }^{10}$. Los EE MM tienen que proporcionar estos indicadores comunes dos veces al año, en torno al 28 de febrero y en torno al 31 de agosto. La CE, en un siguiente paso, actualizará el Scoreboard en abril y octubre, respectivamente.

El primer conjunto sustantivo de información se publicará en febrero de 2022. A partir de esta fecha se podrá comenzar a valorar la suficiencia y adecuación de la información publicada para que tanto las autoridades competentes como los expertos, y la sociedad en su conjunto, puedan monitorizar la implementación de los planes y evaluar su impacto económico y social.

\section{La implementación del NGEU en 2021}

Todos los países presentaron sus PRR en 2021, primer paso para acceder a los fondos, salvo los Países Bajos, debido a retrasos en la formación del nuevo gobierno ${ }^{11}$. La CE dio el visto bueno a 22 pla-

\footnotetext{
${ }^{8}$ Véase https://ec.europa.eu/economy_finance/recovery-and-resiliencescoreboard/index.html

9 Véase Delegated Regulation 2021/2105. https://ec.europa.eu/ economy finance/recovery-and-resilience-scoreboard/index.html

10 Véase Delegated Regulation 2021/2106. https://eur-lex.europa. eu/legal-content/EN/TXT/?uri=uriserv\%3AOJ.L_.2021.429.01.0083.01.

ENG\&toc=OJ\%3AL\%3A2021\%3A429\%3ATOC

11 Los EE MM fueron invitados a presentar sus PRR antes del 30 de abril de 2021, pero pueden hacerlo en cualquier momento hasta mediados de 2022
}

nes, que también fueron aprobados por el Consejo, desbloqueando así los primeros desembolsos correspondientes a la prefinanciación. La CE y el Consejo están en proceso de aprobar la mayoría de los planes de recuperación pendientes, con la excepción de los de Hungría y Polonia. De esta forma, ya se efectuaron los primeros desembolsos correspondientes a la prefinanciación por $56,3 \mathrm{~mm}$ a 21 países (equivalente al $82 \%$ del total de los países que han presentado los planes). Adicionalmente, se desembolsaron $10 \mathrm{~mm}$ correspondientes al primer pago efectuado a España por el cumplimiento de los hitos y objetivos establecidos en su plan de recuperación y resiliencia para 2021. Cerca del $90 \%$ de los pagos efectuados hasta la fecha corresponden a las cuatro grandes economías de la zona euro: España (19 mm), Francia $(5,1 \mathrm{~mm})$, Italia $(24,9 \mathrm{~mm})$ y Alemania $(2,25 \mathrm{~mm})$, más Grecia (4 mm) y Portugal $(2,2 \mathrm{~mm})$, lo que equivale al $93 \%$ del total de los pagos que este grupo de países tenía previsto recibir en 2021 (véase Figura 6). El monto de las transferencias solicitadas por los cuatro planes aún no aprobados asciende a $41 \mathrm{~mm}$ (un $12 \%$ del total).

Para financiar los desembolsos a los EE MM, en 2021 la CE realizó seis transacciones por las que recaudó $71 \mathrm{~mm}$ de euros, que se complementan con letras a corto plazo por las cuales la CE recaudó en 2021 alrededor de $22 \mathrm{~mm}$ de euros ${ }^{12}$.

\section{Condicionantes de la capacidad transformadora del NGEU}

\section{El tamaño del NGEU}

La aprobación del NGEU supuso un importante hito en la respuesta europea, en la medida en la que se respondía a una crisis de naturaleza exógena, pero con un impacto desigual, con un elemento de solidaridad entre países. Este permitía compensar en el corto

\footnotetext{
12 Véase NGEU tracker para más detalle y actualizaciones: https://www. ngeutracker.org/borrowing-operations
} 


\section{FIGURA 6}

\section{CALENDARIO DE PAGOS: AGREGADO SIMPLIFICADO DE LA ZONA EURO}

(En miles de millones de euros)

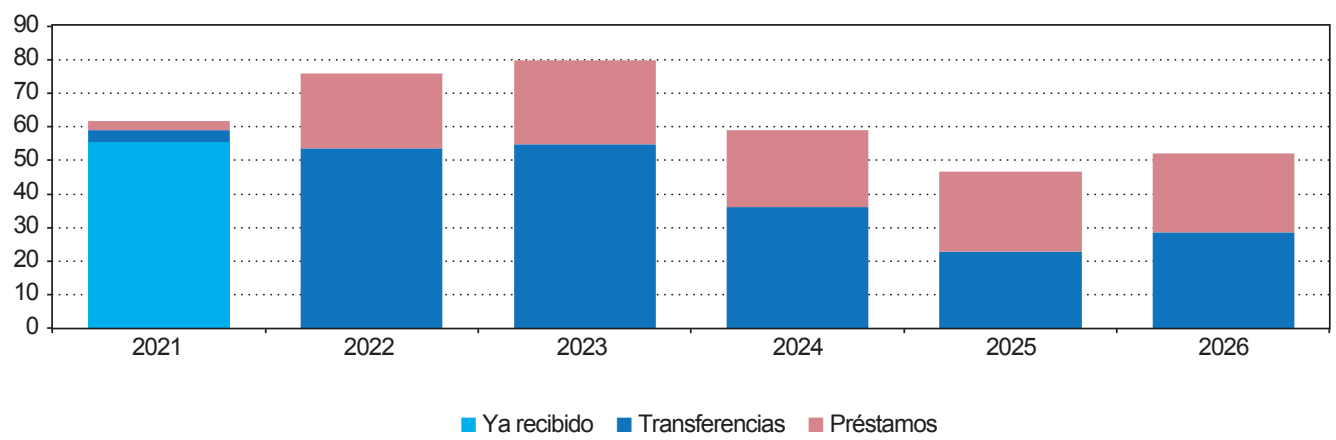

NOTA: Esta figura muestra el calendario anual de pagos (en miles de millones de euros) a realizar por la Comisión Europea para el acumulado de los cuatro grandes de la zona euro (Alemania, España, Francia e Italia) más Portugal y Grecia según los planes de recuperación y resiliencia nacionales.

FUENTE: Elaboración propia a partir de Comisión Europea (2021c).

plazo la caída de la demanda y, de modo más relevante, aumentar el potencial de crecimiento de la economía europea a medio y largo plazo, a través de un impulso reformador y de una reasignación de los recursos productivos hacia áreas como la digitalización o la lucha contra el cambio climático.

Sin embargo, el NGEU se diseñó en un contexto en el que las previsiones económicas proyectaban una fuerte caída del PIB para 2020 pero al mismo tiempo una recuperación de la actividad económica muy robusta relativamente homogénea entre países. En concreto, en el momento de su aprobación, las previsiones proyectaban para la zona euro en 2021 una caída del PIB con respecto a su nivel anterior a la pandemia de $330 \mathrm{~mm}$ de euros (una cifra similar al montante total de transferencias del MRR), frente a una pérdida de $312 \mathrm{~mm}$ de euros que proyectan las últimas previsiones. Sin embargo, no todos los países se han visto igualmente afectados y, mientras que para el conjunto de la Unión Económica y Monetaria (UEM) y para algunos países las últimas previsiones indican una pérdida de PIB ligeramente menor, en otros casos la pérdida de PIB ha sido mucho mayor de lo que las previsiones mostraban en verano de 2020, como se ha comentado anteriormente. En general, hay una mayor dispersión en las pérdidas acumuladas dentro de la UEM de las que se estimaba en el momento de elaboración y calibración del instrumento (véase Figura 7).

La Figura 8 muestra el porcentaje de pérdida de PIB acumulado y las transferencias previstas (como porcentaje del PIB), por país. Se observa una gran heterogeneidad y poca relación entre la pérdida de actividad y el volumen de los fondos. Así, los países del este de Europa son los principales beneficiados de las asignaciones del NGEU, debido a su menor PIB per cápita, aunque la caída de la actividad ha sido comparativamente menor. Por el contrario, la caída en los países del sur de Europa ha resultado más persistente, como consecuencia, entre otros factores, del mayor peso de los servicios relacionados con el turismo.

En todo caso, la utilización del NGEU como herramienta de estabilización macroeconómica no es 
FIGURA 7

\section{CAÍDA ACUMULADA DEL PRODUCTO INTERIOR BRUTO (2020-2021) Y DISPERSIÓN DE LA PÉRDIDA ACUMULADA SEGÚN LAS PREVISIONES DE LA CE PUBLICADAS EN DISTINTAS FECHAS \\ (En \%)}

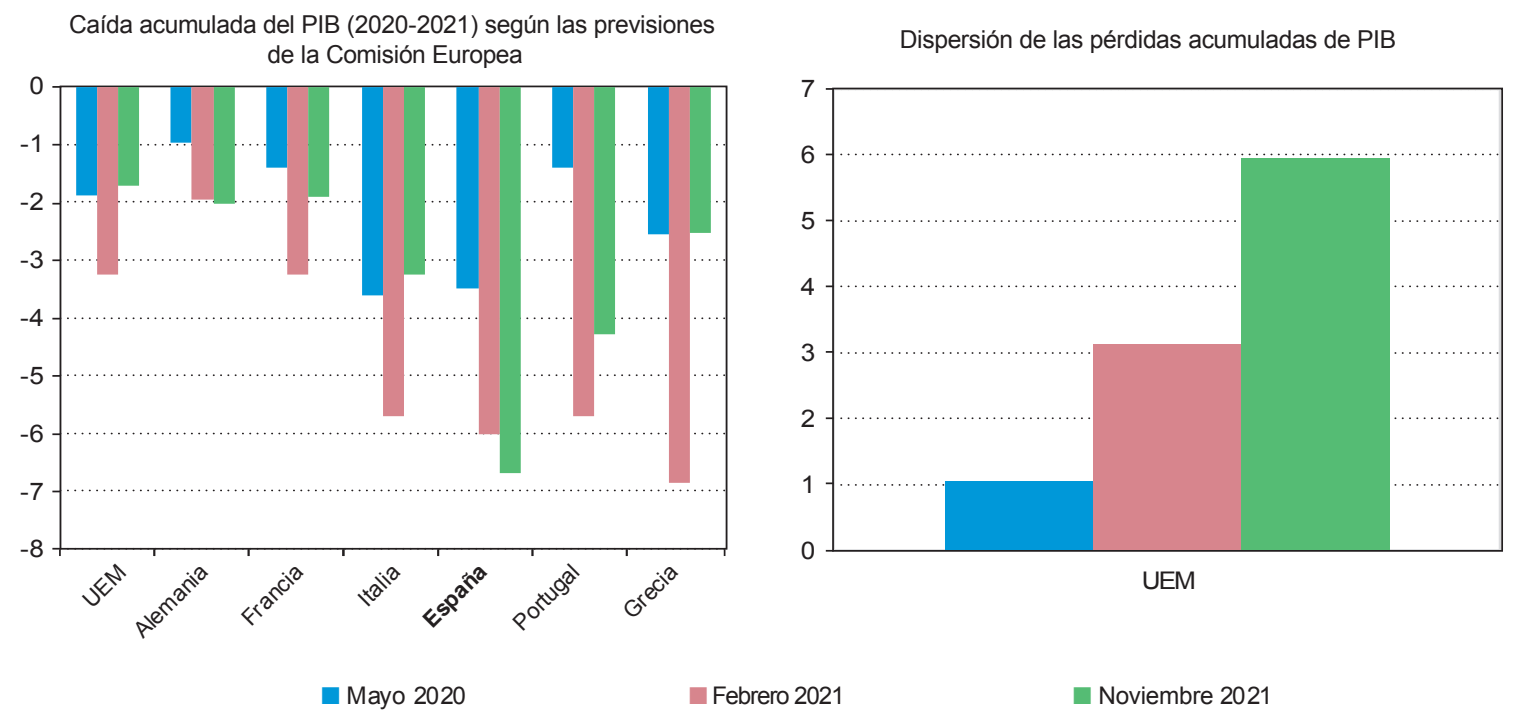

NOTA: El panel de la izquierda muestra la caída acumulada del PIB en 2020 y 2021 según las previsiones de la Comisión Europea (mayo de 2020 , febrero y noviembre de 2021). El panel de la derecha muestra la dispersión en la zona euro de la caída del PIB medida como la desviación típica de la caída acumulada del PIB de todos los países de la eurozona según las diferentes previsiones de la Comisión Europea. FUENTE: Elaboración propia a partir de Eurostat.

inmune a las limitaciones con la que se encuentra la inversión pública en general para realizar esta labor, debido a los retrasos en su implementación (véanse De Jong et al., 2017; Argimón et al., 1994). En este caso, el efecto económico del NGEU, aprobado en una situación de fuerte recesión, no se reflejaría hasta que las economías estuvieran en una trayectoria de recuperación, lo que reduciría su impacto macroeconómico.

\section{Contribución a expandir el volumen de activos seguros}

La financiación del NGEU con emisión a gran escala de deuda pública supranacional supone un paso sin precedentes. Al recaudar tales cantidades en los mercados, la UE se está convirtiendo rápidamente en un actor importante en los mercados de capitales y en un importante proveedor de activos seguros en euros, reforzando así el papel internacional del euro. Si se utiliza por completo el endeudamiento permitido en el NGEU, la deuda supranacional europea se doblaría ${ }^{13}$ (véase Figura 9), otorgando a los inversores internacionales más oportunidades de construir su cartera en la base de activos seguros, diversificados y con una rentabilidad más atractiva que la de otros activos seguros denominados en euros. Además, al emitir el $30 \%$ de la financiación de NGEU $(250 \mathrm{~mm}$ de euros) como bonos verdes, la UE gestionará el esquema de bonos verdes más grande del mundo.

\footnotetext{
13 Véase Delgado-Téllez et al. (2020).
} 


\section{FIGURA 8}

\section{CAÍDA ACUMULADA DEL PIB (2020-2021) Y TRANSFERENCIAS ESTIMADAS DEL MECANISMO DE RECUPERACIÓN Y RESILIENCIA}

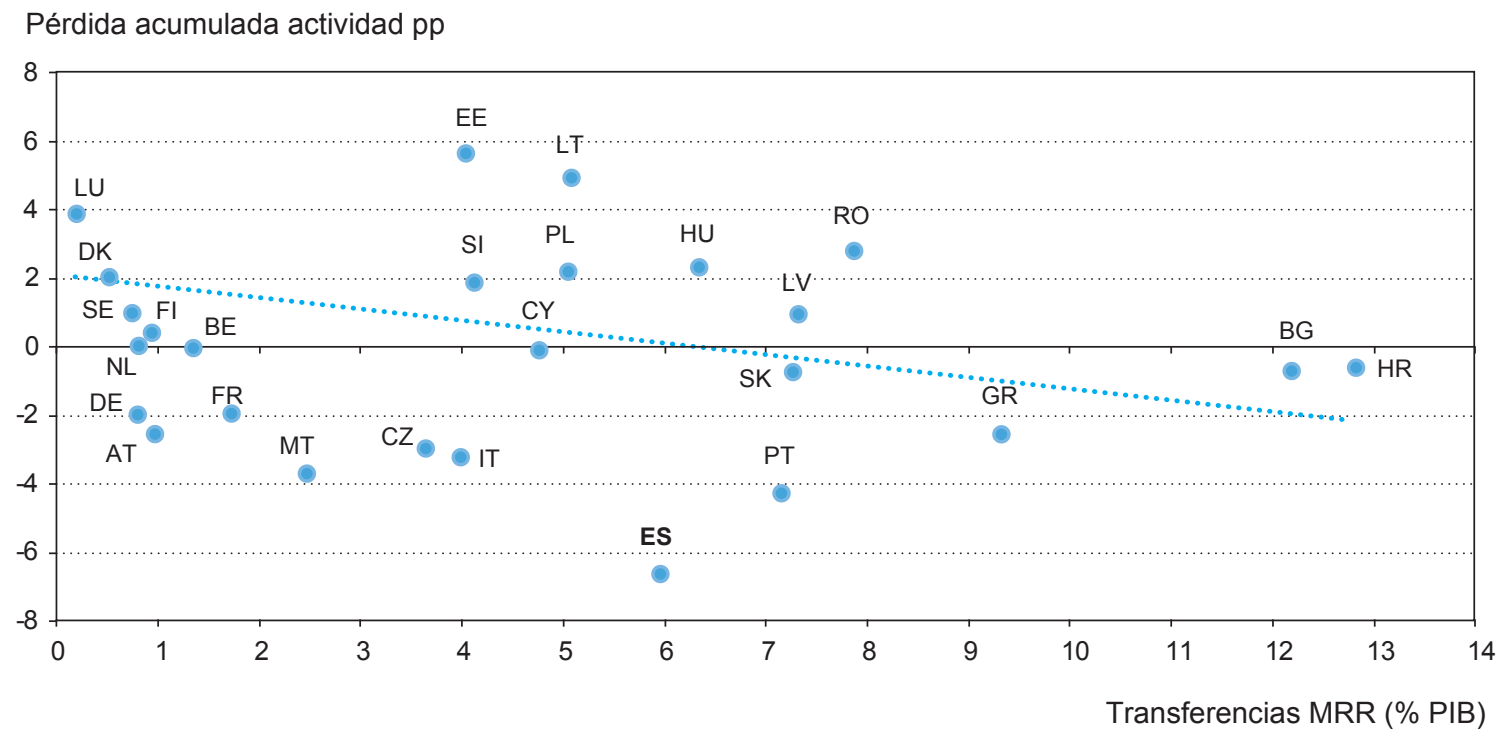

NOTA: Esta figura muestra en el eje horizontal las transferencias estimadas (en \% del PIB) de acuerdo a las últimas previsiones de la Comisión Europea (noviembre de 2021) y en el eje vertical la caída del PIB (2020-2021) también según las últimas previsiones de la Comisión.

FUENTE: Banco de España a partir de Eurostat y Comisión Europea (2021a).

Esta emisión a gran escala es un primer paso para impulsar un mercado suficientemente líquido de activos comunes en euros. Asimismo, la experiencia histórica señala que los avances en el marco institucional europeo contribuyen a reducir la fragmentación financiera en la zona euro. La aprobación del NGEU tuvo efectos positivos en los mercados bursátiles de la eurozona y supuso, asimismo, una relajación en las condiciones de financiación de la deuda soberana de los países de la UEM (Banco de España, 2020a). La evidencia disponible señala que los efectos positivos sobre los mercados financieros de las iniciativas destinadas a una mayor integración europea son significativos (Kataryniuk et al., 2021), en la medida en que se perciben como una garantía de continuidad y fortalecimiento del proyecto europeo, lo que beneficia a los emisores nacionales en euros.

\section{Impacto económico y condicionantes}

Como se ha mencionado repetidamente a lo largo del artículo, el NGEU se diseñó para ayudar a las economías de la UE a recuperarse de la pandemia y estimular el crecimiento a medio y largo plazo, fomentando la resistencia a las crisis e impulsando el potencial económico. Según una primera aproximación tentativa de la CE (Pfeiffer et al., 2021), utilizando el modelo QUEST, el NGEU puede impulsar el PIB real de la UE en el rango de 1,2 a 1,5 puntos porcentuales. El impacto sería mayor en los países del sur de Europa, acercándose a los 2,5 pp en Italia, Portugal y España y superando los $3 \mathrm{pp}$ en Grecia en el momento de mayor impacto, que coincide con el último año (2026) de implementación del programa (véase Figura 10). Cabe destacar que incluso los 
FIGURA 9

STOCK DE ACTIVOS SEGUROS PANEUROPEOS, 2019

(En \% del PIB de la UE-27)

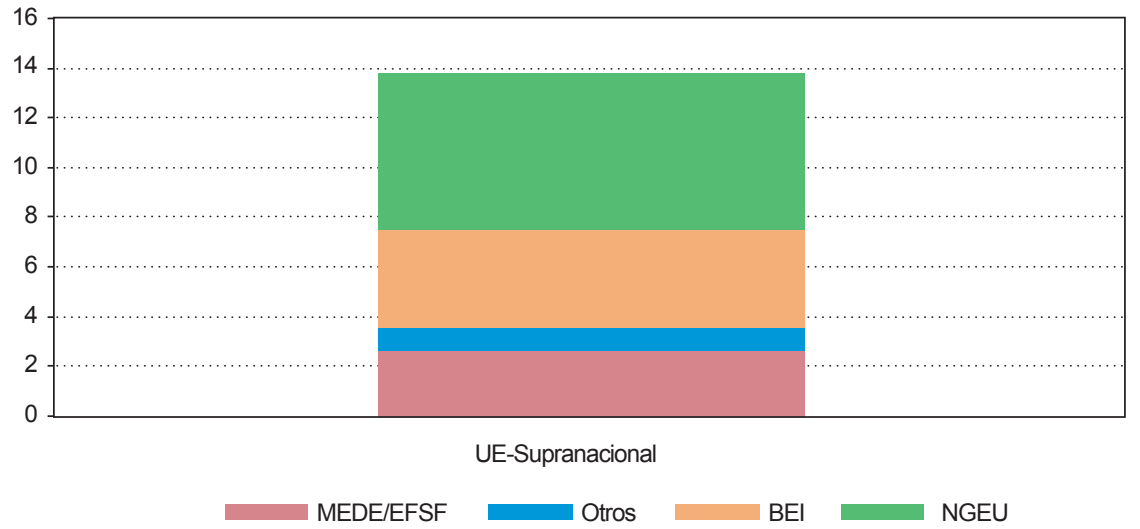

NOTA: EFSF = European Financial Stability Facility; BEI = Banco Europeo de Inversiones

FUENTE: Elaboración propia.

países que reciben proporcionalmente menos ayuda del plan, como Francia o Alemania, experimentan un fuerte crecimiento, beneficiándose en gran parte de efectos de spillover derivados de las conexiones comerciales entre los EE MM (Alloza et al., 2019).

En cualquier caso, existen dudas acerca de la capacidad de la UE para producir tal impacto en el crecimiento a corto o largo plazo. Algunos estudios han señalado que es difícil asegurar la «adicionalidad» de los fondos (Gros, 2021): los gobiernos pueden argumentar en los PRR que las transferencias de la UE financiarán la inversión pública, pero la cuestión es si esta inversión es adicional y genera más gasto o si no lo es, en cuyo caso el dinero de la UE financiaría inversiones que se habrían realizado de todos modos. En este último caso, el gobierno puede utilizar el dinero ahorrado gracias a la redistribución de la inversión ya sea hacia gastos corrientes, para recortar impuestos, o para reducir el déficit presupuestario y la deuda pública.
Otro tema muy debatido es el de la absorción. Se trata de la capacidad tanto de la economía como de la Administración para hacer frente al impulso inversor. Gros (2021) sostiene que las transferencias del MRR representan una parte muy pequeña del gasto público (nacional) pero una parte muy significativa de la inversión pública (nacional). Para los países que reciben una alta proporción de transferencias del MRR como Bulgaria, Portugal, Croacia, España y Grecia, su inversión pública anual se doblaría durante los próximos cuatro a seis años bajo el supuesto de una tasa de absorción del $100 \%$ y adicionalidad total (Alcidi \& Gros, 2020).

Diversos factores pueden hacer cambiar el multiplicador fiscal de los fondos $\mathrm{y}$, por tanto, variar su impacto sobre la economía. En este sentido, el Banco de España (2020b) recoge la importancia de tener en cuenta el grado de complementariedad entre la inversión pública y la inversión privada. Además, el estímulo en economías con más holgura, como España e Italia, tiende a tener multiplicadores más grandes. Finalmente, 
FIGURA 10

IMPACTO DEL NGEU SOBRE EL PIB REAL

(\% de desviación del nivel del PIB real sin NGEU)
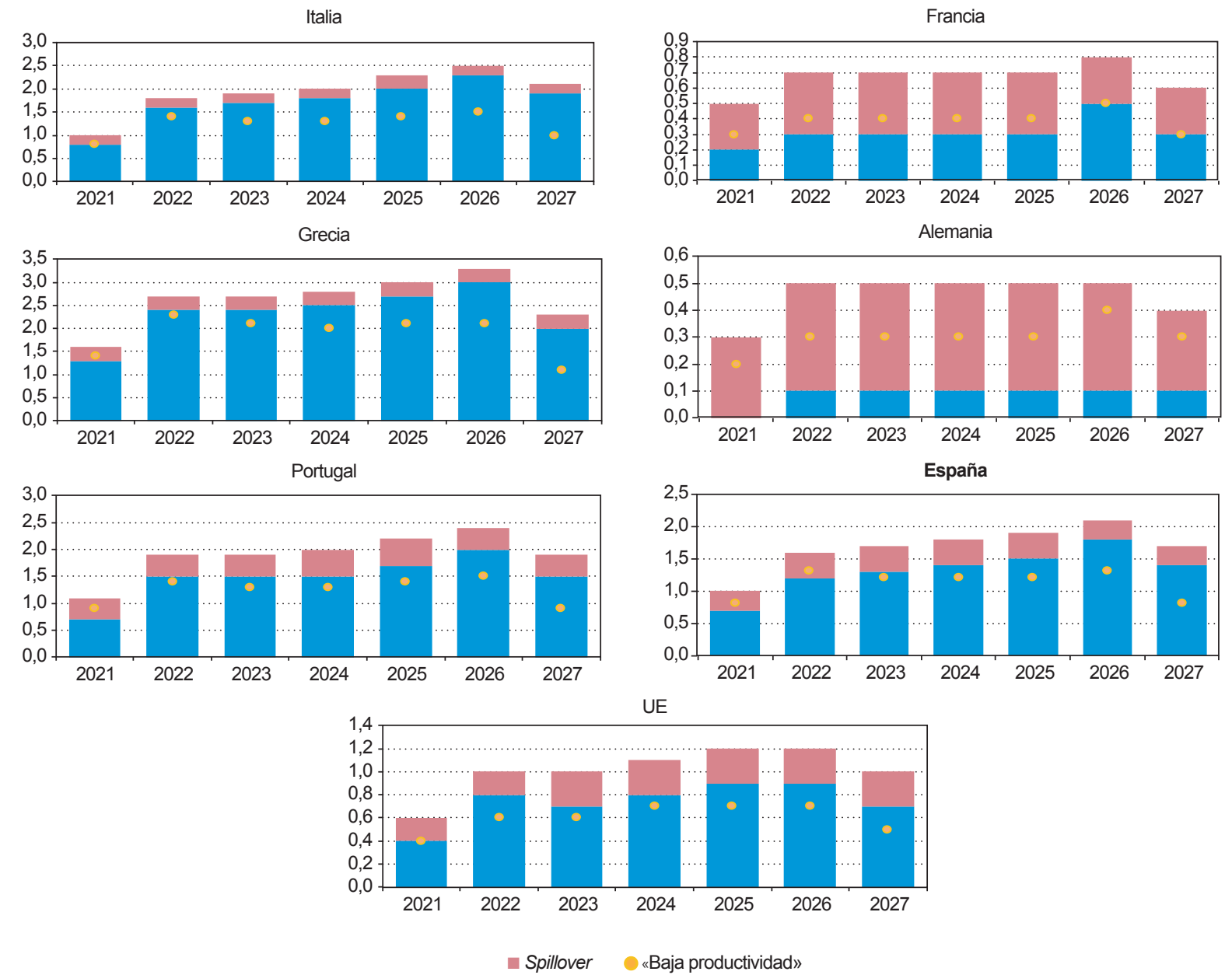

NOTA: 1) Esta figura muestra el impacto estimado de las transferencias y préstamos solicitados del NGEU (MRR) sobre el PIB de los países durante el período de vigencia del programa bajo tres escenarios: el escenario central, que solo tiene en cuenta el efecto directo; el escenario spillover, que añade el efecto indirecto a través de los vínculos comerciales; y el escenario de «baja productividad», que considera una elasticidad significativamente más baja del rendimiento del capital público. En todos los casos se asumen desembolsos lineales durante los seis años de vigencia del programa. 2) Simulaciones realizadas por la Comisión Europea con el modelo QUEST. FUENTE: Pfeiffer et al. (2021).

el impacto del estímulo fiscal es mayor cuando las tasas de interés se encuentran alrededor de su límite inferior efectivo. Por último, aunque todos los países dedican una parte de los fondos a proyectos transnacionales, la gran mayoría de proyectos se fijan por prioridades nacionales por los Estados miembros y en un plazo muy limitado de tiempo. Esto implica que no necesariamente los fondos internalizarán las prioridades de la UE a medio plazo (interconexión, estrategia verde), no generando los spillovers más potentes y pudiendo derivar 
en que la asignación sectorial no sea la más adecuada. Además, esta clave nacional de los planes de recuperación, puede derivar en competencia entre países e ineficiencias en el uso de los fondos, así como cuellos de botella en algunos materiales críticos. En concreto, las inversiones relacionadas con las transiciones digital y ecológica son muy intensivas en determinados insumos, que se encuentran muy concentrados geográficamente, lo que reduciría el impacto del plan debido al fuerte contenido importador necesario para su ejecución y al previsible aumento de precios derivado de la escasez de materiales (Boer et al., 2021).

En el largo plazo, el impacto positivo más importante del NGEU puede provenir de las reformas estructurales y de la interacción entre las reformas y la inversión. La evidencia empírica reciente señala que las reformas que reducen las fricciones en el mercado laboral y en el de productos pueden potenciar el efecto de los fondos europeos (Albrizio \& Geli, 2021). Además, algunas reformas, en particular en la Administración pública, la planificación, la justicia y el entorno empresarial, están diseñadas para alentar la inversión del sector privado.

\section{¿Uso de los préstamos?}

Como se ha señalado anteriormente, solo siete países han solicitado hasta el momento hacer uso de los préstamos del MRR. Esto contrasta con la evidencia de otros instrumentos aprobados en el contexto de la pandemia. Así, los préstamos otorgados por el SURE con características similares, fueron solicitados por un total de 19 países. La principal razón de esta divergencia puede descansar en que el SURE financiaba gastos ya incurridos, en concreto, aquellos destinados a los esquemas de apoyo al empleo temporal, como los Expedientes de Regulación Temporal de Empleo (ERTE). Sin embargo, los préstamos del MRR deben destinarse a nuevas inversiones y reformas, lo que podría alterar la trayectoria de deuda pública de los países. Además, en la medida en la que el SURE podría ser interpretado como el primer paso hacia un sistema de reaseguro de desempleo permanente (Vandenbroucke et al., 2020), esto podría haber incentivado su uso por parte de los países favorables a su institucionalización.

En este sentido, es posible que los países agoten el margen con el que cuentan para solicitar nuevos préstamos. Como se explica en Burriel et al. (2022), si bien estos préstamos generan una cantidad de ahorros limitada en situaciones en las que las primas de riesgo están comprimidas, los ahorros crecen exponencialmente en el caso de que estas crezcan. En todo caso, la optimalidad del uso de los préstamos dependerá crucialmente de una cuidadosa selección de los proyectos a acometer con esos fondos, que permita aumentar el crecimiento en el largo plazo y así minimizar el eventual efecto negativo de un mayor endeudamiento público. Por ello, sería conveniente aumentar el período de disponibilidad de estos préstamos (mediante la extensión del período de petición o mediante la constitución de fondos de inversión al efecto a nivel nacional), para permitir una evaluación cuidadosa de las inversiones realizadas y no discontinuar aquellas con rendimientos positivos.

\section{Conclusiones}

EI NGEU supone un paso significativo en el proceso de integración europea ya que ha contribuido a mitigar, al menos temporalmente, dos carencias fundamentales del encaje institucional europeo: la falta de elementos fiscales estabilizadores supranacionales y la escasez relativa de activos seguros en euros. Mirando hacia delante, obviamente, estas dos necesidades son estructurales para el correcto devenir de la Unión Económica y Monetaria europea y, por tanto, el éxito del NGEU debería contribuir a la creación de un estado generalizado de opinión entre los EE MM para el afianzamiento de estructuras permanentes que permitiera cubrirlas.

La falta de precedentes de programas de esta ambición, no obstante, hace que exista una elevada incertidumbre sobre el impacto global del NGEU. En 
concreto, su efecto final dependerá de una serie de factores condicionantes: capacidad de implementación siguiendo el calendario fijado; eficiencia de dicha implementación; si el tamaño aprobado, decidido de manera arbitraria, será suficiente para cumplir los objetivos; si la distribución de fondos entre países, heterogénea, permitirá evitar la divergencia entre las economías más afectadas por la crisis y el resto; si se producirán impactos sobre el crecimiento potencial de la UE y sus economías nacionales, o solo se tratará de un estímulo de demanda transitorio.

Las respuestas a estas preguntas no se conocerán hasta que no haya pasado un cierto tiempo. En el corto plazo, para poder ir anticipando las respuestas y permitir a las autoridades reaccionar, será necesario un esfuerzo significativo de transparencia por parte de las distintas Administraciones públicas, en particular, en lo relativo a la publicación y diseminación de toda la información necesaria, para que los expertos, y la sociedad en su conjunto, puedan monitorizar la implementación de los planes y evaluar su impacto económico y social.

\section{Referencias bibliográficas}

Albrizio, S. \& Geli, J. F. (2021). Un análisis empírico de los factores que pueden potenciar la efectividad del programa Next Generation EU. Boletín Económico del Banco de España n. ${ }^{\circ}$ 4/2021. Artículos Analíticos.

Alcidi, C. \& Gros, D. (2020). Next Generation EU: A Large Common Response to the COVID-19 Crisis. Intereconomics, 55(4), 202-203.

Alloza, M., Burriel, P. \& Pérez, J. J. (2019). Fiscal policies in the euro area: revisiting the size of spillovers. Journal of Macroeconomics, 61, 103132.

Arce, Ó. J., Kataryniuk, I., Marín, P. \& Pérez, J. J. (2020). Reflexiones sobre el diseño de un fondo de recuperación europeo. Documentos Ocasionales del Banco de España n. ${ }^{\circ} 2014$.

Argimón, I., González Páramo, J. M., Martín, M. J. \& Roldán, J. M. (1994). Productividad e infraestructuras en la economía española. Documentos de Trabajo del Banco de España n. ${ }^{\circ} 9313$.

Banco de España. (2020a). Next Generation EU: Main characteristics and impact of its announcement on financial conditions. Quarterly report on the Spanish economy, Economic Bulletin, 3.

Banco de España. (2020b). Recuadro 5.2. El papel de la inversión pública a escala europea en la recuperación económica. Informe Anual 2019.

Boer, L., Pescatori, A., Stuermer, M. \& Valckx, N. (2021, November 10). Soaring Metal Prices May Delay Energy Transition. IMF, International Monetary Fund Blog.

Bruegel. (2021). Los planes de Recuperación y Resiliencia de los países de la Unión Europea. https://www.bruegel.org/ publications/datasets/european-union-countries-recoveryand-resilience-plans/

Burriel, P., Kataryniuk, I. \& Pérez, J. J. (2022). An extended debt sustainability assessment tool for euro area sovereigns, with an application to EU loans. Próximo a aparecer, Documentos Ocasionales del Banco de España.

Comisión Europea. (2020a). El Fondo REACT-EU. https:// ec.europa.eu/regional_policy/en/newsroom/coronavirusresponse/react-eu

Comisión Europea. (2020b). Mecanismo de Recuperación y Resiliencia - Asignación de subvenciones por Estado Miembro. https://ec.europa.eu/info/files/recovery-andresilience-facility-grants-allocation-member-state_en

Comisión Europea. (2020c). Semestre Europeo 2020: las Recomendaciones específicas de país. https:// ec.europa.eu/info/publications/2020-european-semestercountry-specific-recommendations-commissionrecommendations en

Comisión Europea. (2021a). El Mecanismo de Recuperación y Resiliencia. https://ec.europa.eu/info/business-economyeuro/recovery-coronavirus/recovery-and-resiliencefacility_en\#national-recovery-and-resilience-plans

Comisión Europea. (2021b). Planes de Recuperación y Resiliencia nacionales. https://ec.europa.eu/info/businesseconomy-euro/recovery-coronavirus/recovery-andresilience-facility_en\#national-recovery-and-resilience-plansr

Comision Europea. (2021c). Evaluación de los Planes de Recuperación y Resiliencia nacionales. https://ec.europa. eu/info/business-economy-euro/recovery-coronavirus/ recovery-and-resilience-facility_en\#national-recovery-andresilience-plans

D'Alfonso, A., Delivorias, A., Milotay, N. \& Sapala, M. (2021, January). Economic and Budgetary Outlook for the European Union 2021. EPRS, European Parliamentary Research Service, PE 679.062.

De Jong, J., Ferdinandusse, M., Funda, J. \& Vetlov, I. (2017). The effect of public investment in Europe: a model based assessment. Documentos de Trabajo del Banco Central Europeo n. ${ }^{\circ} 2021$.

Delgado-Téllez, M., Kataryniuk, I., López Vicente, F. \& Pérez, J. J. (2020). Endeudamiento supranacional y necesidades 
de financiación en la Unión Europea. Documentos Ocasionales del Banco de España n. 2021.

García Perea, P., Millaruelo de la Fuente, A., Mora Bajén, V. M. \& Sánchez Carretero, C. (2020). El Semestre Europeo 2020 y las recomendaciones específicas para España. Boletín Económico del Banco de España $\mathrm{n}^{\circ} 3$.

Gros, D. (2021, May 19). Next Generation EU: applying some basic economic principles [Executive education course on the EU Recovery Plan]. School of Transnational Governance, European University Institute, Florence. https://www.eui.eu/ past-courses?id=the-recovery-plan-for-europe\#people

Kataryniuk, I., Mora-Bajén, V. M. \& Pérez, J. J. (2021). EMU deepening and sovereign debt spreads: using political space to achieve policy space. Documentos de Trabajo del Banco de España n. ${ }^{\circ} 2103$.
Pfeiffer, P., Varga, J. \& in't Veld, J. (2021, July 15). Quantifying Spillovers of Next Generation EU Investment. EC, European Commission. DG ECFIN, Discussion Paper No. 144.

Unión Europea. (2021). Reglamento (UE) 2021/241 del Parlamento Europeo y del Consejo de 12 de febrero de 2021 por el que se establece el Mecanismo de Recuperación y Resiliencia. Diario Oficial de la Unión Europea. https://eur-lex.europa.eu/legal-content/ES/TXT/ HTML/?uri=CELEX:32021R0241\&from=EN

Vandenbroucke, F., Andor, L., Beetsma, R., Burgoon, B., Fischer, G., Kuhn, T., Luigjes, C. \& Nicoli, F. (2020). The European Commission's SURE initiative and euro area unemployment re-insurance. Vox CEPR Policy Portal. https://voxeu.org/ article/european-commission-s-sure-initiative-and-euro-areaunemployment-re-insurance 
ANEXO

TABLA 1

RECOMENDACIONES ESPECÍFICAS POR PAÍSES EN EL AÑO 2020

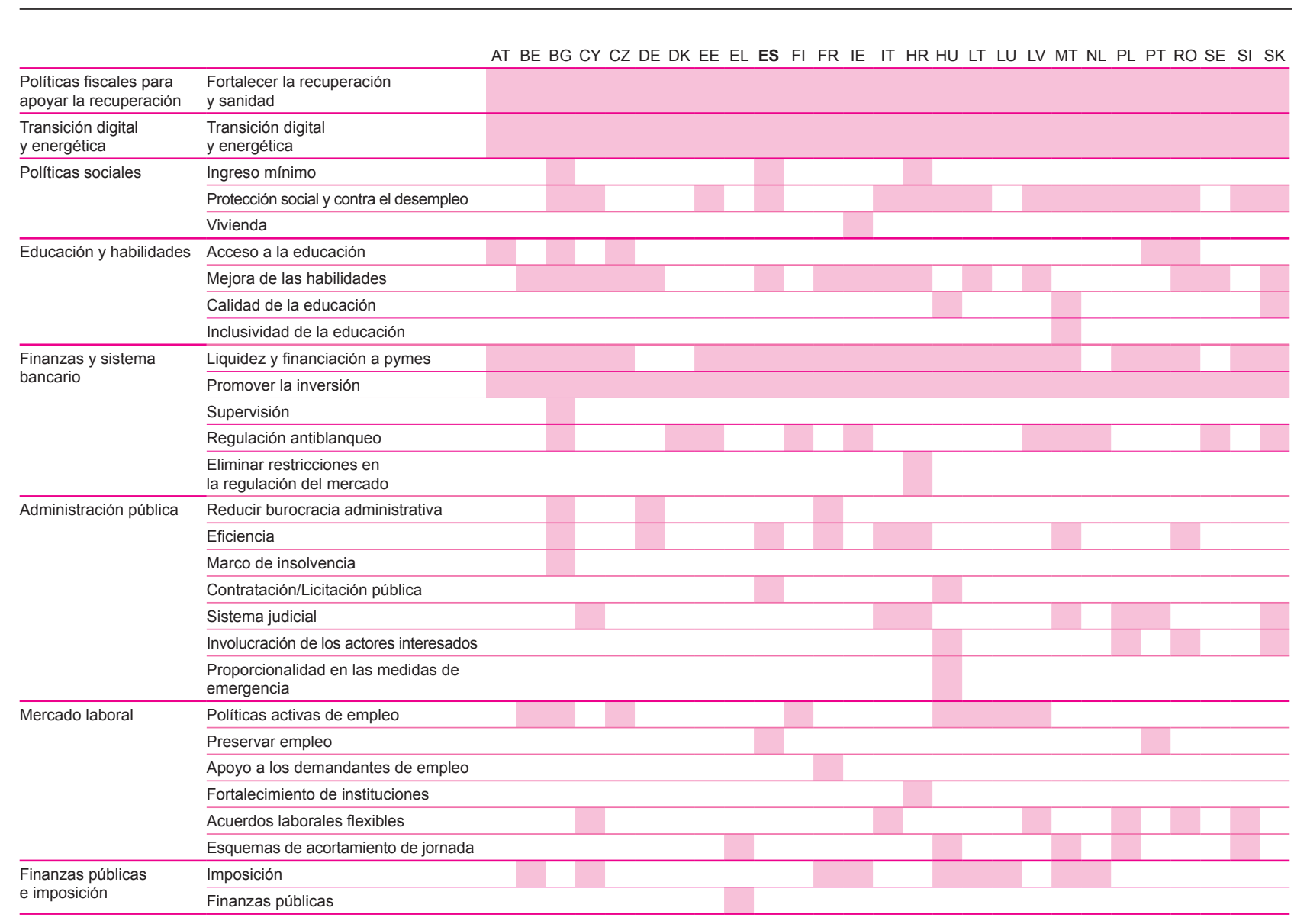

NOTA: 1) Se resaltan las áreas en las cuales los países han recibido recomendaciones. 2) AT: Austria; BE: Bélgica; BG: Bulgaria; CY: Chipre; CZ: Chequia; DE: Alemania; DK: Dinamarca; EE: Estonia; EL: Grecia; ES: España; FI: Finlandia; FR: Francia; HR: Croacia; HU: Hungría; IE: Irlanda; IT: Italia; LT: Lituania; LU: Luxemburgo; LV: Letonia; MT: Malta; NL: Países Bajos; PL: Polonia; PT: Portugal; RO: Rumanía; SE: Suecia; SI: Eslovenia; SK: Eslovaquia.

FUENTE: Comisión Europea (2020c). 